\title{
SPUNTI PER UNA DESCRIZIONE DEI VERBI IN -EGGIARE E -IZZARE: I DATI DELL'ITALIANO ANTICO IN PROSPETTIVA DIACRONICA E COMPARATIVA
}

\author{
Liana TRONCI \\ Università per Stranieri di Siena
}

\begin{abstract}
It): L'articolo propone una descrizione dei derivati verbali in -eggiare e -izzare dell'italiano antico, in prospettiva sincronica e diacronica. La ricerca è corpus-based e si basa sullo spoglio delle ricorrenze dei 240 lemmi in -eggiare e degli 85 lemmi in -izzare attestati nel TLIO. I due suffissi sono entrambi di origine latina: -eggiare è esito del regolare mutamento fonetico di verbi formati con il suffisso latino tardo -idiāre, mentre -izzare è forma dotta, perfettamente corrispondente al latino classico -izäre. In sincronia, i derivati in -eggiare e-izzare presentano molti casi di allotropia, indice della sovrapposizione funzionale dei due suffissi. In diacronia, i dati dell'italiano antico, comparati con quelli del greco antico, permettono di gettare nuova luce sulla situazione latina che, oltre al ridotto grado di produttività lessicale dei suffissi, ne documenta i derivati solo in testi dalla forte connotazione alloglotta.
\end{abstract}

\begin{abstract}
En): This article deals with verbal forms suffixed with -eggiare and -izzare in Old Italian. Relevant forms were collected from the TLIO lexical and textual resources: they amount to 240 -eggiare items and 85 -izzare items. Both suffixes derive from Latin: the former is the output of regular morpho-phonetic changes from Late Latin -idiāre, whereas the latter occurs in loanwords and calques formed on Classical Latin verbs in -izäre. Synchronically, there are several allotropes, indicating the functional overlapping of the two suffixes. Diachronically, the comparison with Ancient Greek data allows us to shed a new light on a state of affairs in Latin usage and to account for constraints imposed on lexical productivity and textual distribution of relevant forms in Latin.
\end{abstract}

Parole-chiave (It): derivazione verbale; italiano antico; ricerca su corpus; diacronia latinoromanza

Keywords (En): verbal derivation; Old Italian; corpus-based research; Latin-Romance diachrony

\section{Introduzione}

Questo studio propone un'analisi corpus-based dei derivati verbali in -eggiare e -izzare in italiano antico. Le due classi di derivati sono entrambe produttive, seppur con qualche differenza, nell'italiano odierno (cf. DARDANO, 2009: 47-48, 53-54); semanticamente, esse si presentano in distribuzione complementare: i derivati in -eggiare "sono di solito intransitivi ed esprimono l'azione o il modo di essere o di comportarsi implicati dalla base, talora con sfumatura intensiva o frequentativa", mentre quelli in -izzare "sono di solito transitivi ed hanno valore causativo, indicando un processo di trasformazione o di assimilazione".

I suffissi -eggiare e -izzare derivano da uno stesso suffisso latino, che presenta uno statuto foneticamente variabile: -issārel-izāre/-idiāre sono le tre varianti del suffisso, che ricorrono per es. nelle forme matrissāre 'comportarsi da madre', formato su mater, matris 'madre', betizāre 'esser molle come una barbabietola', dal

\footnotetext{
${ }^{1}$ Si cita dal Vocabolario Treccani della lingua italiana disponibile on-line, s.v. -eggiare e -izzare rispettivamente.
} 
nome beta 'barbabietola' e gargaridiāre 'fare gargarismi', prestito dal greco

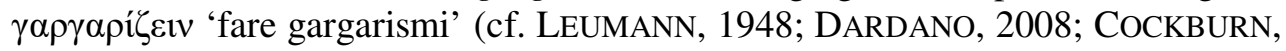
2012; TRONCI, 2017 per un quadro della situazione latina). Le tre forme del suffisso sono attestate in momenti diversi della storia del latino: la variazione è certamente da mettere in relazione con l'origine alloglotta delle forme pertinenti, che, almeno nelle loro prime attestazioni, sono parole di prestito o calco di verbi greci con suffisso -í̧દıv. ${ }^{2}$ Le forme suffissali italiane -eggi(are) e -izz(are) sono esito di due diversi processi diacronici, rispettivamente regolare mutamento morfo-fonetico e prestito/calco dal latino per via dotta (cf. ROHLFS, 1966: §276). I due processi sono testimoniati anche in altri sistemi romanzi, come diremo più dettagliatamente in seguito ( $\$ 2.4)$.

Obiettivo del lavoro è descrivere i dati dell'italiano antico, in prospettiva sincronica e diacronica. Da un lato, si intende verificare quali fossero i rapporti quantitativi tra i derivati delle due classi e se la differenziazione semantica tra di esse fosse già netta in italiano antico o se vi fossero ancora casi di possibile sovrapposizione; dall'altro, si tenterà di analizzare il rapporto in diacronia tra le prime attestazioni di verbi in -eggiare e -izzare dell'italiano e il sistema latino, per il quale è necessario precisare la questione della produttività delle forme in -issāre/-izāre/-idiāre, anche in relazione alla situazione del greco antico da cui le forme latine originano sotto forma di prestiti e calchi. Lo studio diacronico prevede dunque la descrizione di tre momenti: il greco antico, il latino e l'italiano antico. Per i primi due, ci si avvarrà degli studi che sono stati condotti sul tema, mentre per i dati dell'italiano antico si è proceduto alla raccolta delle entrate lessicali del dizionario Tesoro della Lingua Italiana delle Origini e allo spoglio delle attestazioni presenti per ciascun lemma nelle banche dati del Corpus OVI dell'italiano antico. ${ }^{3}$ Quantitativamente, si sono così ottenuti 240 lemmi in -eggiare e 85 lemmi in izzare, per un totale di 325 lemmi complessivi. ${ }^{4}$ In Appendice al presente lavoro sono riportati gli elenchi in ordine alfabetico delle forme pertinenti.

Un primo utile esempio dell' ampio spettro di variazione di entrambe le classi derivazionali, per quanto riguarda sia il tipo di basi lessicali implicate (nomi, aggettivi, verbi, avverbi), sia le funzioni sintattico-semantiche dei derivati, è fornito dagli es. (1)-(2), dove si osservano, in presenza del medesimo suffisso -eggiare,

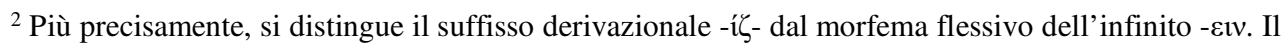
suffisso - $i \zeta$ - non è ereditato, ma nasce da coalescenza tra la consonante finale di basi lessicali terminanti in - $1 \delta-/-1 \gamma$ - (come $\dot{\varepsilon} \lambda \pi \imath \delta-, \sigma \alpha \lambda \pi \imath \gamma(\gamma)$-) ed il suffisso di derivazione indoeuropea *-je/o-, molto produttivo nella formazione di verbi denominali e deaggettivali. Il morfema derivazionale $-i \zeta$ - nasce dunque da ri-segmentazione di forme verbali denominali e deaggettivali in *-je/o- (cf. TRONCI, 2017: 82ss. per una discussione di questi aspetti).

${ }^{3}$ A partire dalla pagina http://tlio.ovi.cnr.it/TLIO/ sono consultabili sia il dizionario sia i testi. Le edizioni dei testi da cui si traggono gli esempi sono quelle del corpus del TLIO.

${ }^{4} \mathrm{I}$ lemmi attestati in documenti di aree italo-romanze non toscane non saranno presi in considerazione, se non marginalmente, in questo lavoro. Si tratta, nello specifico, delle forme terminanti in -ezar, -izar di area settentrionale e dei derivati in -iare, -iari di area meridionale e siciliana, rispettivamente. Si rinvia a RoHLFs (1966: §§277-278), TEKAVČIĆ (1972: III, 119) e ZAMBONI (1980-1981) per gli esiti del nesso latino <di> + vocale, Meul (2013: cap. 6) per il particolare caso dell'evoluzione nel ladino dolomitico, Giuliani (2019: 194-198) per alcune forme lessicali dell'area italo-romanza di ambito marinaresco.
} 
derivati con valori transitivi di tipo causativo e derivati con valori intransitivi di tipo eventivo. Per il primo tipo, il verbo francheggiare 'rendere franco', formato sull'aggettivo franco: le due attestazioni in (1a) e (1b) esemplificano i due valori transitivi lessicalizzati 'rassicurare' e 'liberare'. Per il secondo tipo, il verbo biancheggiare 'diventare bianco, risplendere' dall'aggettivo bianco nell'es. (2).

(1a) se non che coscienza m'assicura la buona compagnia che l'uom francheggia sotto l'asbergo del sentirsi pura (Divina Commedia, Inferno XXVIII 115-117)

(1b) immantanente mandò per quello uomo e francheggiollo da ogne prigione e trebuto

(2) I' mossi i piè del loco dov'io stava, (Leggenda Aurea 27) per avvisar da presso un'altra istoria, che di dietro a Micòl mi biancheggiava (Divina Commedia, Purgatorio X 70-72)

Tale aspetto, come avremo modo di argomentare, differenzia il sistema dell' italiano antico da quello odierno, in cui la distinzione semantica tra le due classi di derivati pare più netta.

L'articolo è strutturato come segue. Nel §2 si descrivono i verbi latini in -issāre/ -izārel-idiāre e i loro rapporti nel diasistema latino e nella diacronia del latino, con un breve resoconto bibliografico relativo ai processi che hanno determinato la loro comparsa nei diversi sistemi romanzi. Nel $\$ 3$ si analizzano le forme dell'italiano antico, con particolare attenzione alla differenziazione semantica tra le due classi e ai casi di allotropia. Nel $\S 4$ si propongono osservazioni comparative e diacroniche sulle tre varietà interessate: greco antico, latino e italiano. Infine nel $\$ 5$ si traggono alcune conclusioni.

\section{Dal latino al romanzo}

\subsection{Il suffisso -eggiare}

Come si è già anticipato, i suffissi italiani -eggiare e -izzare sono esito di due diversi processi fono-morfologici. Da un lato, -eggiare presuppone la forma del suffisso latino -idiāre, attestata nella documentazione solo a partire dal I millennio d.C. e presumibilmente marcata come variante diastraticamente bassa di -izāre. Un esempio della distribuzione sociolinguisticamente determinata delle due forme è fornito dalla coppia baptidiāre 'inzuppare' vs baptizāre 'battezzare', attestate, la prima, variante bassa, in Apicio e, la seconda, variante alta, nella letteratura cristiana. Della pronuncia palatalizzata della sequenza $<\mathrm{di}>+$ vocale del latino, rappresentabile grosso modo foneticamente come [gj] e diffusasi presumibilmente a partire dal II sec. d.C., l'affricata palatale sonora geminata [dd]] del suffisso -eggiare rappresenta la naturale continuazione nel romanzo di area toscana (ROHLFS, 1966: §276). Le forme in -eggiare dell'italiano antico sono quindi sorte per regolare mutamento fonetico dal latino, a partire dalla variante diastraticamente bassa e popolare del suffisso (presumibilmente più frequente nella lingua orale di quanto non lo testimoni la documentazione scritta), per poi diffondersi nel lessico dell'italiano antico tramite un processo di rianalisi morfologica. A titolo di esempio, si consideri il verbo italiano ormeggiare, voce appartenente al lessico marinaresco, 
attestata tanto in testi toscani quanto in testi settentrionali (cf. TLIO, s.u. ormeggiare; GIULIANI, 2019: 194). L'origine di tale forma è stata individuata in una voce tardo-latina non attestata *hormidiāre, presupposta dai lessicografi sulla base

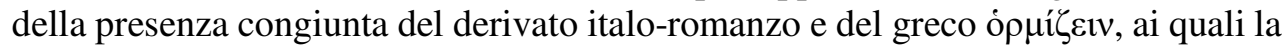

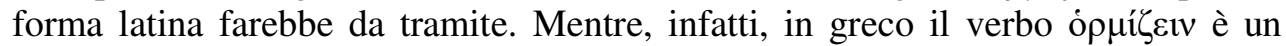

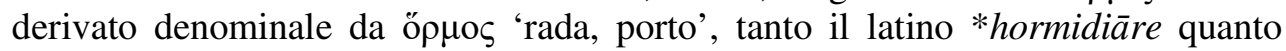
l'italiano ormeggiare non sono forme motivate e trasparenti nei due sistemi linguistici: la forma latina è un prestito dal greco e quella italiana è esito di regolare mutamento morfo-fonetico dal latino. ${ }^{5}$ Un percorso in parte simile pare aver seguito il verbo citarizzare/citareggiare 'suonare la cetra', i cui allomorfi hanno diversa distribuzione diatopica: la forma citareggiare, lemmatizzata nel TLIO nella variante fonetica toscana, è attestata solo in varietà settentrionali e presuppone una forma latina non attestata *citharidiāre, a fianco dell'attestata citharizäre, che è invece continuata come voce dotta nella variante toscana citarizzare.

\subsection{Il suffisso -izzare}

Veniamo ora al suffisso -izzare, più complesso da spiegare rispetto a -eggiare. Da una parte, infatti, -izzare è presente soprattutto in parole di origine cólta, per es. anatematizzare, metaforizzare, tesaurizzare, o in registri tecnici di lingua, per es. aromatizzare, cauterizzare, clisterizzare, senapizzare per quello della medicina, fatto che lascia presupporre che i verbi in -izzare siano in gran parte prestiti di verbi latini in -izāre in italiano e che la forma fonetica del suffisso [id'dza:re] con l'affricata alveolare sonora geminata sia dovuta a semplici processi di integrazione delle originarie forme latine con grafema $\langle\mathrm{z}>$, presumibilmente realizzato in latino come fricativa alveolare sonora [z]. Già nel sistema latino, del resto, la variante del suffisso -izāre rappresenta la forma per così dire "normalizzata", almeno graficamente, che sostituì a partire dall'anno 81 a.C. la forma -issāre, in seguito all'introduzione del grafema $<\mathrm{z}>$ nell'alfabeto latino. ${ }^{6}$ Prima di tale riforma, l'integrazione di prestiti greci in -í̧દıv si dà solo nelle forme latine in -issāre, come

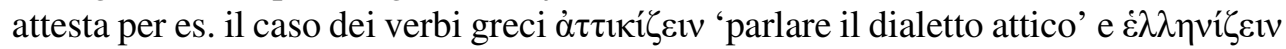
'parlare greco', penetrati in latino sotto forma, rispettivamente, di prestito (atticissāre) o calco (graecissāre) e presenti nella seguente attestazione plautina, in combinazione con la formazione squisitamente latina sicilissāre $e^{7}$ 'parlare il dialetto siciliano', neologismo plautino modellato sulle forme di derivazione greca:

\footnotetext{
${ }^{5}$ Dall'italiano ormeggiare il francese avrebbe preso in prestito ormeger e, per tramite del veneziano armizàr, la forma sarebbe passata anche in serbocroato e in greco moderno, cf. VIDOS (1939: 496499).

${ }^{6}$ Sulla questione si rinvia a Arena (1965), Mignot (1969: 330-339), Biville (1990: 99-136), DARDANO (2008). Per una sintesi TRONCI (2017). In prospettiva romanza cf. AlKIRE, Rosen (2010: 308).

${ }^{7}$ Secondo alcune edizioni la forma è sicilicissitāre, con un aggettivo latino non attestato *sicilicus,

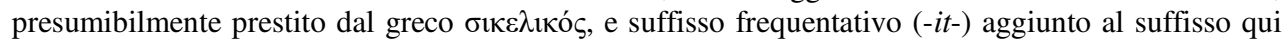
pertinente-iss-it-āre.
} 
(3) atque adeo hoc argumentum graecissat: tamen

non atticissat, uerum sicilissat (Plauto Menecmi 11-12)

'e dunque questa commedia parla greco: non (greco) attico però, ma (greco) siciliano, 8

La riforma grafica si rese necessaria, evidentemente, proprio per trascrivere il suono $[\mathrm{z}]$ corrispondente al grafema greco $\langle\zeta>$ e presente in molti prestiti dal greco, forme in -í̧દıv incluse. Tornando al suffisso italiano -izzare, la trafila dotta non è tuttavia la sola possibile. ROHLFS (1966: §276) osserva, infatti, che il nesso latino $<\mathrm{di}>+$ vocale, presente anche nel suffisso -idiāre, offre in area toscana due diversi esiti: a quello con l'affricata palatale sonora geminata [dd]] si affianca quello con l'affricata alveolare sonora geminata [ddz] in parole come mèzzo < lat. MEDIUS, rozzo < lat. *RUDIUS, mozzo 'parte della ruota' < lat. MODIUS, razzo 'raggio della ruota' < lat. RADIUS, e nei verbi qui pertinenti in -izzare. ${ }^{9}$ Se per mèzzo si potrebbe anche supporre una derivazione semicolta, come del resto è generalmente proposto per i verbi in -izzare, molto più difficile è accogliere tale proposta per le altre parole, che designano chiaramente strumenti e attitudini della vita popolare. Dato che l'esito [ddz] del nesso latino $<\mathrm{di}>+$ vocale è di tipo settentrionale, ROHLFS avanza l'ipotesi che le parole mozzo e razzo di origine settentrionale siano penetrate in toscano in un'epoca in cui "l'industria dei carri toscana ha subito l'influsso settentrionale" (p. 391) e che anche mèzzo e rozzo abbiano avuto la stessa sorte. L'estensione dell'ipotesi ad includere le forme in -izzare è possibile, ma in tal caso rimane da spiegare il diverso trattamento della vocale iniziale $<i>$ che si mantiene in -izzare, a differenza dell'esito regolare [e] che si trova in tutta l'area italoromanza settentrionale, sia nelle forme toscanizzate in -ezzare, per es. battezzare < lat. baptidiāre, olezzare < lat. *olidiāre, sia in quelle dialettali, per es. il lombardo antico brutezar, il ligure antico lampezar etc. (cf. RoHLFS, 1969: §1160). ${ }^{10}$ Senza assumere una posizione definitiva al riguardo, ci si limita a sottolineare che, quanto alle forme dell'italiano antico, la diversa trafila popolare e dotta delle forme in -eggiare e -izzare è plausibile, anche in considerazione del fatto che nella maggior parte dei casi le forme in -izzare hanno effettivamente una forma latina in -izäre corrispondente, per es. allegorizāre, anathematizāre, aromatizāre, cauterizāre, hymnizāre, inthronizāre, pulverizāre, scandalizāre, thesaurizāre, caso che si dà solo raramente per le forme in -eggiare, che sono per lo più neoformazioni romanze.

\footnotetext{
${ }^{8}$ Una traduzione meno banalizzante dei verbi in -issāre del passo plautino lascerebbe implicito il riferimento alla lingua (parlare greco, attico o siciliano), che qui è peraltro sicuramente pertinente, per una soluzione più ammiccante e indeterminata con i verbi corrispondenti italiani in -eggiare (grecheggiare, atticheggiare e sicilianeggiare), che, seppur non presenti nei lessici, sono senz'altro plausibili e comprensibili al lettore.

${ }^{9}$ Le confusione grafica tra $<\mathrm{z}>\mathrm{e}<\mathrm{di}>+\mathrm{V}$-, documentata dalle iscrizioni tardo-latine, per es. ZANUARIU per IANUARIU (CIL X 2466), ZIACONUS per DIACONUS (CIL III 8652), OZE per HODIE (CIL VIII 8424) nonché da testimonianze dei grammatici (Isidoro di Siviglia nel VII sec. d.C. scrive solent Itali dicere ozie pro hodie 'gli italiani dicono di solito ozie al posto di hodie'), segnala una qualche confusione tra i due suoni: cf. ALKIRE, ROSEN (2000: 61) che concludono: "[t]his use of $\langle\mathrm{z}>$, a letter borrowed from Greek, is probably meant to represent the newly affricating $/ \mathrm{j} /$ "'.

${ }^{10}$ Con l'eccezione delle forme del veneziano che presentano la vocale [i] e la deaffricazione dell'affricata seguente, realizzata come fricativa alveolare sonora [z], per es. lampizàr 'lampeggiare', manizàr 'maneggiare', negrizàr 'annerire', tonizàr 'tuonare' (RoHLFS, 1969: §1160).
} 


\subsection{I “doppioni” in -eggiare/-izzare del corpus}

Nel lemmario del TLIO sono presenti alcuni "doppioni" in -eggiarel-izzare, cui merita dedicare una breve discussione. Oltre al tipo colpeggiare/colafizzare "tirare colpi, percuotere', trasparente il primo come derivato dal nome colpo, non analizzabile il secondo, in quanto prestito dal latino colaphizāre, a sua volta prestito

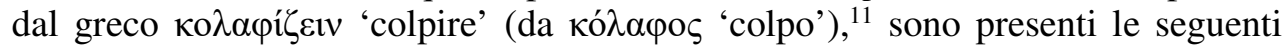
coppie, formate ciascuna da una medesima base lessicale: allegoreggiare/allegorizzare (allegoria), barbareggiare/barbarizzare (barbaro), citareggiare/citarizzare $($ citara $=$ cetra $)$, favoleggiarelfavolizzare (favola), festeggiarelfestizzare (festa), filosofeggiarelfilosofizzare (filosofo), mercanteggiare Imercantizzare (mercante), salmeggiare/salmizzare (salmo), solenneggiare /solennizzare (solenne), tiranneggiareltirannizzare (tiranno).

La presenza di tali coppie si spiega variamente. Nella prima allegoreggiare/allegorizzare, il lemma in -eggiare è presupposto sulla base di un' unica attestazione nel Capitolo a compendio della Divina Commedia di Busone de' Raffaelli da Gubbio (XIII-XIV sec.), mentre il lemma in -izzare risulta attestato in testi toscani e settentrionali. Anche nella coppia citareggiare/citarizzare, il primo lemma è integrato sulla base di un' attestazione di area settentrionale (bolognese per la precisione, nei Commenti al Purgatorio di Jacopo Della Lana) che riferisce di una forma citariçando:

(4) Dinanzi parea gente, çoè la compagnía de David ch'andavano cantando e citariçando (Jacopo della Lana, Commenti al Purgatorio X)

Così anche per la coppia salmeggiare/salmizzare, nella quale il lemma salmeggiare è la forma toscanizzata del veneziano salmizàr attestato unicamente nel testo anonimo del XIV sec. noto come Navigatio Sancti Brendani. Diverso è il caso di barbareggiare/barbarizzare entrambi attestati una sola volta, in un testo di area toscana, il primo, e con riferimento alla lingua: : già la loro lingua barbareggiava per estraneità, e in un testo di area veronese, il secondo, con soggetto umano: acioché nelo proferire l'omo non barbarizi. Per quanto entrambi usati intransitivamente, i due verbi non hanno la medesima distribuzione e lo stesso spettro semantico: con riferimento alla lingua, barbareggiare alterna con 'essere barbaro' o 'suonare barbaro' mentre barbarizzare con soggetto umano può essere sostituito anche da 'comportarsi da barbaro' (cf. §3).

Così pare anche il caso della coppia filosofeggiarelfilosofizzare, entrambi attestati una sola volta nel corpus del TLIO, in due contesti molto diversi: uno intransitivo, con il verbo filosofeggiare parafrasabile con l'espressione 'atteggiarsi a filosofo', e uno passivo, in cui filosofizzare vale grosso modo 'riempire di argomentazioni filosofiche':

\footnotetext{
${ }^{11}$ Che colafizzare fosse forma non più trasparente è chiaramente rivelato dalla seguente attestazione, in cui il verbo è glossato: il quale me colafizza, cioè mi percuote nel collo, onde colafizzare è percuotere nel collo si legge nella Esposizione di Salmi di Ranieri de' Rinaldeschi (p. 118 dell'ed. Bini).
} 
(5a) la fortuna vuole ch'io più speditamente filosofegi (Ottimo Commento della Commedia, Inferno IV)

(5b) ciascuno nostro parlamento debbe essere umile, non enfiato, non retoricato, sillogizzato, non filosofizzato (Giovanni Dominici, Libro d'amore di carità)

Vi sono poi casi nei quali una delle due forme della coppia ha rare attestazioni (talvolta è un hápax legómenon) a fronte delle numerose attestazioni dell'altra, senza che tra le due forme vi siano differenze nella distribuzione diatopica. È il caso di favolizzare e festizzare, entrambi attestati una sola volta (es. 6a-6b), rispetto a favoleggiare e festeggiare, entrambe forme ancora vitali nell'italiano odierno.

(6a) nel qual segno [Gemini], favolizzando, dicono che furono convertiti li figliuoli di Leda, cioè furono Castore e Polluce (Ottimo Commento della Commedia, Paradiso XXVII)

(6b) quando sarete stati in terra a voi inimica, lei festizzerà e riposerassi nelle feste della sua solitudine (Bibbia volgare secondo la rara edizione del I ottobre MCCCCLXXI, Levitico XXVI)

Vi sono poi casi in cui le due forme della coppia appaiono perfettamente intercambiabili dal punto di vista sintattico-semantico: per es. i verbi signoreggiare/signorizzare ricorrono entrambi in contesti tanto transitivi (es. 7a-7b) quanto intransitivi (es. 8a-8b), ma il primo membro della coppia è attestato più anticamente del secondo (metà XIII sec. rispetto a metà XIV sec.) ed ha una frequenza di gran lunga maggiore in italiano antico (più di 900 ricorrenze nel TLIO per signoreggiare e solo 9 per signorizzare). Dei due lemmi, del resto, signoreggiare è quello ancora oggi in uso.

(7a) Ma poi c'Amore si fece sentire dentro dal cor signoreggiar la gente

(Pier delle Vigne, Però c'Amore no si pò vedere, vv. 5-6)

(7b) volendo signorizzare lo mondo, e giudicar le genti

(Domenico Cavalca, Esposizione del Simbolo degli Apostoli, I 23)

(8a) E volendo i Cerchi signoreggiare, furono signoreggiati (Dino Compagni Cronica I 27)

(8b) Nella sua virtù signorizza egli sempre

(Bibbia volgare secondo la rara edizione del I ottobre MCCCCLXXI, Salmi LXV v. 5)

Una simile allotropia si osserva anche nella coppia solenneggiare/solennizzare, entrambi attestati nel valore 'celebrare con solennità, festeggiare solennemente':

(9a) Apo li antichi, li principj de' mesi si guardavano, e solenneggiavano

(Ottimo Commento della Commedia, Purgatorio XVI)

(9b) La sagra de la Chiesa tra l'altre festivitadi è molto solennizzata da la Chiesa

(Leggenda Aurea 81)

Dai casi di allomorfia e/o allotropia appena osservati si può avanzare l'ipotesi che in italiano antico la distribuzione dei due suffissi in funzione dei valori sintattico-semantici del derivato non fosse così netta come è invece nell'italiano odierno, dove -izzare e -eggiare sono orientati rispettivamente verso l'espressione di funzioni transitivo-causative e intransitivo-eventive (DARDANO, 2009: 47-48, 53- 
54). Le oscillazioni tra una forma e l'altra del suffisso erano plausibilmente dovute anche alla polimorfia diatopica dei suffissi. Le forme in -izàr del veneziano, per esempio, foneticamente suonavano molto simili a quelle in -izzare del toscano - ed è anche possibile che come tali siano state toscanizzate in alcuni casi - ma, a differenza di queste, provenivano da regolare mutamento fonetico dal latino ed erano quindi forme di derivazione non dotta. Per es. il verbo tiranizàr, attestato nell' anonimo Commento morale in versi delle favole attribuite a Walterius, è sì il corrispondente veneziano della forma tiranneggiare, documentata sia in area toscana sia in testi settentrionali e meridionali, ma foneticamente è più prossima a tirannizzare, e non si può quindi escludere che tale forma fosse presente nella lingua a livello di uso e/o di sistema.

\subsection{Un rapido sguardo alle altre varietà romanze}

L'italiano non è la sola lingua nella quale la classe latina in -issāre/-izārel-idiāre si è continuata in due distinte serie di derivati verbali, differenziate sia nella forma sia nella funzione. In francese, si hanno i verbi in -oyer e -iser, di derivazione fonetica regolare e non produttiva, la prima, di provenienza dotta e produttiva, la seconda, per es. foudroyer 'fulminare' e étatiser 'nazionalizzare'. Si rinvia a GUILBERT (1971: XXXIII-XXXIV), ZINK (1986: 95) e al Trésor de la Langue Française informatisé (TLFi) s.u. -oyer e -iser per ulteriori dettagli. ${ }^{12}$ In spagnolo, le due classi, rispettivamente in -ear e-izar, sono entrambe ad elevata produttività e presentano una diffenziazione semantica dei derivati comparabile a quella dell'italiano: per es. blanquear 'biancheggiare' e carbonizar 'carbonizzare' (cf. ALVAR, POTTIER, 1983: §311; RAINER, 1993: 458-465, 592-596; PHARIES, 2002: 184-186, 373-374; Bergua CAVERO, 2004: 185; COCKBURN, 2012, 2013). Anche in rumeno sono presenti due suffissi: $-e z a$, non produttivo e derivato da regolare mutamento morfo-fonetico dal latino, ricorre per es. in boteza 'battezzare', e -izal-isa sorti da rianalisi di prestiti dal latino, dal francese, dall'italiano e dall'inglese (cf. GoICU, 2007). Per un inquadramento del problema in una prospettiva romanza generale si rinvia a ZAMBONI (1980-81) che illustra a grandi linee il fenomeno - proprio di alcune varietà romanze, tra cui il rumeno e alcuni dialetti italo-romanzi - in base al quale il suffisso derivazionale latino è stato integrato nella flessione verbale. Qui non di discuterà di tale processo, che tuttavia è indice dell'importanza dei suffissi considerati per tutta l'area romanza, e si rinvia agli studi di MEUL $(2009,2013)$, che offre un'analisi approfondita della situazione del ladino dolomitico, anche in confronto con altre varietà che presentano il medesimo fenomeno. ${ }^{13}$

\footnotetext{
${ }^{12}$ Disponibile al sito http://atilf.atilf.fr/tlf.htm (ultimo accesso novembre 2019).

13 "Whereas the lexicalized occurrence of remnants of the segment -IDI- can be considered a 'panRomance' phenomenon, its inflectionally-bound application is much less 'widespread' in the Romance territory: [...] Romanian is the only Romance standard language in which the vestiges of -IDI- occur in a non-lexicalized configuration. [...] next to Romanian, the inflectional -IDI-pattern figures in a handful of Italian and Rhaeto-Romance dialect varieties, with possible (at least 'functionally') ramifications to Gallo-Romance" (MeUL, 2013: 74).
} 


\section{I dati dell'italiano antico: processi derivazionali e valori sintattico-semantici}

\subsection{La derivazione verbale: breve excursus bibliografico}

Il tentativo di classificazione più completo dei derivati verbali dell'italiano è stato proposto da GROSSMANN (2004: 450ss.), che considera le tre classi più produttive nella formazione di verbi denominali e deaggettivali in italiano, cioè $\mathrm{i}$ verbi in -eggi(are), -izz(are) e -ific(are). Come denominali, "[i] derivati in -eggirappresentano circa il $47 \%$ dei verbi denominali suffissati, ma a giudicare dal numero relativamente basso delle neoformazioni, circa il 19\% dei verbi denominali suffissati attestati dopo gli anni ' 50 , la produttività del suffisso non sembra molto alta" (p. 450); come deaggettivali, "[i] derivati in -eggi- rappresentano circa il $21 \%$ dei verbi deaggettivali suffissati; le neoformazioni sono relativamente poche, costituiscono solo il $2 \%$ dei verbi deaggettivali suffissati attestati dopo gli anni ' 50 " (p. 459). Per quanto riguarda i derivati denominali in -izz(are), essi "rappresentano circa il $40 \%$ dei verbi denominali suffissati e, a giudicare dal numero relativamente alto delle neoformazioni, circa il $73 \%$ dei verbi denominali suffissati attestati dopo gli anni "50, la produttività del suffisso sembra piuttosto alta" (p. 451); i deaggettivali con lo stesso suffisso "rappresentano circa il $70 \%$ dei verbi deaggettivali suffissati e, a giudicare dal numero elevato delle neoformazioni, circa il $94 \%$ dei verbi deaggettivali suffissati attestati dopo gli anni ' 50 , la produttività del suffisso sembra molto alta" (p. 460).

La studiosa separa i denominali dai deaggettivali e propone una classificazione di tipo, potremmo dire, top down. Per i denominali fa uso di parametri di diverso tipo: (a) la classe azionale del predicato ${ }^{14}$, (b) i tratti $[ \pm$ animato $]$ e $[ \pm$ umano] relativi al soggetto, (c) la classe nozionale del nome che fa da base lessicale ( $\mathrm{N}$ locativo, $\mathrm{N}$ di strumento etc.). Ottiene così un numero elevato di classi distinte, talvolta ulteriormente articolate al loro interno, che non sono specifiche di un tipo di derivazione, e quindi di un suffisso, ma presentano esponenti appartenenti ai diversi tipi derivazionali: per es. nella classe che designa eventi telici parafrasabili come "«(far) diventare (come un) N», dove la base nominale indica lo stato-meta: X diverrà $\mathrm{o}$ sarà fatto divenire $\mathrm{N} \mathrm{o}$ simile $\mathrm{a} \mathrm{N}$, acquisirà o gli saranno fatte acquisire le qualità di N ovvero delle qualità simili a N" (p. 454) troviamo forme delle tre classi: idoleggiare, mostrificare e demonizzare.

Altrettanto articolata è la classificazione dei derivati deaggettivali secondo la quale (p. 459ss.), i verbi in -eggi(are), -izz(are) e -ific(are) sono di tipo incoativo "[s]e il referente del soggetto dell'enunciato è affetto, indipendentemente dalla propria volontà, da un mutamento di proprietà" (p. 461), causativo "se invece ne è causa intenzionale" (p. 461), stativo o continuativo se "designano un'azione non telica" (p. 462). Ovviamente, anche in questo caso, gioca un ruolo importante il tipo di proprietà designata dall'aggettivo che fa da base lessicale al derivato (p. 462ss.).

Questa pur rapida discussione mostra con chiarezza che la complessità lessicosintattica dei derivati oggetto di questo studio è molto difficilmente riconducibile a

\footnotetext{
${ }^{14}$ Secondo il modello vendleriano, che suddivide i verbi in base alle loro proprietà azionali in quattro classi: risultativi (+durativi, +telici), trasformativi (-durativi, +telici), continuativi (+durativi, -telici) e puntuali (-durativi, -telici).
} 
unità. ${ }^{15}$ D'altra parte, la studiosa stessa riconosce che la molteplicità di significati e funzioni cui è associato il derivato dipende dai diversi modi in cui è attivata, nel processo di derivazione, la portata predicativa del nome che fa da base lessicale. Tale attivazione è, peraltro, socialmente e culturalmente determinata: "[i]l verbo denominale suffissato è potenzialmente in grado di designare una grande varietà di situazioni, nelle quali il referente della base potrà avere diversi ruoli”, i quali "possono dipendere dalle attività normalmente associate al referente in una determinata comunità culturale" (GROSSMANN, 2004: 451).

Indipendente dal piano strettamente morfologico (derivati per affissazione, conversione, parasintesi), la questione riguarda più nel profondo i processi funzionali coinvolti nella creazione di un verbo a partire da una base lessicale nominale o aggettivale. Agli aspetti strettamente semantici (rapporto tra significato del derivato rispetto a quello della base lessicale) si associano quelli sintattici: il verbo, per sua natura, ha una cornice argomentale esplicita che, nel nome, può rimanere implicita, per es. negli usi strettamente referenziali. Il processo di "verbificazione" o "verbizzazione" presuppone dunque l'esplicitazione delle proprietà predicative del nome stesso, come afferma anche DARDANO (2009: 46): "[i]l passaggio da $\mathrm{N}$ a V sottintende una frase, costituita da un sintagma nominale soggetto (SN) e da un sintagma verbale (SV)". In altre parole, poiché il processo di formazione di un verbo ha per risultato la designazione di un processo, il lessema che fa da base lessicale al derivato non potrà avervi semplice valore referenziale ma vi entrerà come parte di una struttura predicativa soggiacente. ${ }^{16}$

Nel quadro teorico della Lexical Conceptual Semantics (LCS) (JACKENDOFF, 1991), si sono proposte analisi di derivati verbali affini a quelli qui considerati, come le formazioni verbali inglesi in -ize, classe molto produttiva sorta da rianalisi di prestiti francesi in -iser (LIEBER, 1998, 2004; PLAG, 1999), e i verbi in -ícє1 del greco moderno, anch'essi molto produttivi, che rappresentano la continuazione ininterrotta dei verbi in -í̧દıv del greco antico (CHARITONIDIS, 2007). Come precisa PLAG (1999: 122), il quadro teorico della LCS propone un "output-oriented model of the formation of -ize derivatives, in which the meaning of the derivative results from the interaction of the meaning of the stem with the semantic structure of possible -ize derivatives". Come dice la denominazione stessa, il modello è fortemente dipendente dall'interpretazione semantica dei derivati, cioè dalla parafrasi che qualunque parlante può attribuire al derivato: ospedalizzare è 'mandare in ospedale', bambineggiare è 'comportarsi come un bambino' e via

\footnotetext{
${ }^{15}$ In una prospettiva molto generale, la domanda è posta anche da BAUER (2008: 207): "it is not yet entirely clear whether we can say that 'verbalization' is a single meaning, or whether several types of verbalization, some of them marked by the same affixes, need to be distinguished".

${ }^{16}$ Studi su ambiti specifici delle due classi di derivati sono, per es., LA FAUCI $(2006,2010)$, che analizza i tipi deonomastici rappresentati da catoneggiare 'fare il Catone, comportarsi come Catone' e petrarcheggiare 'imitare (lo stile, l'opera di) Petrarca' come esiti di processi rispettivamente antonomastici e metonimici della base nominale, e GRANDI (2008), che riconosce nei derivati in -eggiare la presenza di un valore alterativo, dovuto alla "spinta convergente di due condizionamenti: da un lato, quello di forme costruite, spesso con funzione caricaturale, su nomi propri; dall'altro, quello di forme in cui la base ha un significato inerentemente negativo [per es. asineggiare, LT]" (p. 23).
} 
dicendo. Rispetto alle possibili parafrasi ${ }^{17}$ determinate per l'insieme dei derivati in -ize dell'inglese, gli studiosi che seguono il modello teorico della LCS forniscono una formalizzazione per mezzo di meta-predicati (CAUSE, GO, BECOME etc.) e macro-ruoli (THEME, PROPERTY, PATH etc.), assunti entrambi come primitivi semantici. Si ha l'impressione che il modello sia una riscrittura, attraverso un'apposita metalingua, dell'interpretazione del parlante. Per tale ragione, non faremo uso di tale modello né della formalizzazione ad esso correlata, pur facendo ricorso, per i fini squisitamente descrittivi di questo lavoro, all'uso di parafrasi. Le considereremo, però, come tali, senza assumere che "there is a form of mental representation, so-called 'conceptual structures', that is common to all languages and that serves as the 'syntax of thought' (JACKENDOFF, 1991: 10)" (PLAG, 1999: 120).

Nel séguito del lavoro si descrivono i derivati in -eggiare (§§3.2-3.4) e -izzare (§3.5), suddivisi in base alla categoria lessicale della base. La produttività della derivazione da nomi e aggettivi e, viceversa, la non produttività della derivazione da verbi e avverbi non stupisce, perché è la medesima situazione osservabile nell'italiano odierno. Stupisce invece l'elevata produttività di -eggiare a fronte di -izzare, i cui derivati presentano rapporti numerici invertiti nell' italiano odierno (cf. GROSSMANN, 2004: 450-451, 459-460).

\subsection{Processi non produttivi: verbi in -eggiare derivati da avverbi e verbi}

Partiamo dai verbi derivati da avverbi. Si tratta di tre sole forme in -eggiare: spesseggiare da spesso 'frequentemente', dietreggiare 'andare dietro' da dietro e indietreggiare da indietro. ${ }^{18}$ Quanto al primo, lo si trova in contesti tanto transitivi ('fare spesso qcs.') quanto intransitivi ('accadere spesso'):

(10a) Le femine di Teba, ammonite per cotali essempli, spesseggiano $i$ nuovi sacrifici, e danno gl'incensi, e onorano le sante altari (Arrigo Simintendi, Metamorfosi d'Ovidio volgarizzate, III)

(10b) Essendo dunque il corpo suo giaciuto molto tempo sotterrato, e spesseggiando li miracoli sanza fine (Leggenda Aurea 108)

I verbi dietreggiare e indietreggiare ricorrono invece in contesti intransitivi, parallelamente al verbo indietreggiare dell'italiano odierno.

La classe dei deverbali è di poco superiore, quanto a numerosità, rispetto alla precedente. ${ }^{19}$ Si tratta in tutti i casi di verbi in -eggiare (non vi sono, infatti, deverbali in -izzare): annulleggiare rispetto ad annullare, avvileggiare rispetto ad

\footnotetext{
${ }^{17}$ Per Plag (1999: 125), che critica le quattro LCS individuate da LIEBER (1998), le LCS soggiacenti ai derivati in -ize sono le sette seguenti: (1) locative $=$ 'put into X' (hospitalize); (2) ornative $=$ 'provide with X' (patinize); (3) causative = 'make (more) X' (randomize); (4) resultative = 'make into X' (peasantize); (5) incoative = 'become X' (aerosolize); (6) performative = perform X' (anthropologize); (7) similative $=$ 'act like $\mathrm{X}$ ' (powellize).

${ }_{18} \mathrm{Vi}$ sono anche le forme lenteggiare 'andar lentamente' (da lento, uso avverbiale dell'aggettivo, parallelo a lentamente) e arratteggiare 'andar velocemente' (da ratto), attestate in uno stesso e unico documento, le Formule notarili aretine del primo Trecento (ed. PIERI, 1972), che riportano: Da guardare è el notaio [e] ei testimoni conosce(n)ti; e a (n)çi le(n)teggia(r)e ch'aratteggiare a fare una carta.

${ }^{19}$ Sui deverbali nell'italiano odierno cf. BERTINETTO (2004: 465-472).
} 
avvilire, beffeggiare rispetto a beffare, calcitreggiare 'prendere a calci' rispetto a calcitrare, brandeggiare rispetto a brandire, leccheggiare rispetto a leccare, lustreggiare rispetto a lustrare, penseggiare rispetto a pensare, pondereggiare rispetto a ponderare, pronteggiare 'riprodurre, raffigurare' rispetto a prontare 'imprimere / prendere l'impronta', scalcheggiare rispetto a scalcare. Nella maggior parte dei casi il derivato in -eggiare presenta valori semantici e impieghi sintattici affini a quelli del verbo che ne fa da base lessicale. Il processo di derivazione si correla quindi in tali casi ad una nuance frequentativa, che il derivato manifesta rispetto al verbo base, per es. penseggiare 'riflettere attentamente, pensare ancora' rispetto a pensare oppure ad una semantica valutativa (cf. BERTINETTO, 2004: 470, GRANDI, 2008: 19). In certi casi, si può anche ipotizzare che la creazione del derivato sia semplicemente dovuta alla ricerca di effetti fonici, per es. nell'unica attestazione di annulleggiare, nelle Rime di Bindo Bonichi: Chi suo voler ben signoreggia / tutto 'Imondo annulleggia, in cui il verbo è in rima con signoreggiare.

\subsection{Verbi in -eggiare deaggettivali}

I verbi deaggettivali in -eggiare sono numerosi e paiono costituire una classe produttiva già in italiano antico. Essi sono creati a partire da aggettivi qualificativi che si riferiscono a proprietà varie. Un primo gruppo, presente anche nell'italiano odierno, è quello dei derivati da aggettivi di colore (biancheggiare, biondeggiare, gialleggiare, rosseggiare, verdeggiare) che presentano il significato intransitivoeventivo 'mostrare il colore X; essere / diventare del colore X'. Nel Decameron (ma anche altrove in Boccaccio) è attestato il verbo verdeggiare (es. 11a), mentre nella Divina Commedia ricorre il verbo rosseggiare, molto attestato in italiano antico, riferito a Marte (es. 11b); il verbo gialleggiare, invece, si trova attestato una sola volta nel corpus del TLIO nel Commento alla Divina Commedia di Francesco da Buti della fine del XIV sec., sempre con riferimento a Marte (es. 11c):

(11a) Quivi s'odono gli uccelletti cantare, veggionvisi verdeggiare i colli e le pianure

(Decameron I, introduzione)

(11b) Ed ecco qual, sorpreso dal mattino, per li grossi vapor Marte rosseggia giù nel ponente sovra'l suol marino (Divina Commedia, Purgatorio II 13-15)

(11c) imperò rosseggia, mentre il sole gialleggia

(Francesco da Buti, Commento alla Divina Commedia, Paradiso XIV)

Con gialleggiare si è presumibilmente di fronte ad un esempio di creazione estemporanea sul modello di rosseggiare: per quanto presente ancora in testi del Novecento (è attestato in D'Annunzio e Gadda, per es., stando alle informazioni fornite dal GDLI, s.u. gialleggiare), la forma appartiene ad un registro di lingua scritto e letterario, diversamente da biancheggiare, rosseggiare o verdeggiare. Infine biondeggiare non è attestato nel corpus del TLIO ma nel Volgarizzamento della Storia della guerra di Troia di Guido delle Colonne, con riferimento al colore dei capelli: Si fissamente ragguardava in lei molti capegli biondeggiare con isplendore rilucente. 
Un secondo gruppo è quello degli aggettivi denotanti dimensioni: grandeggiare attestato in Cecco Angiolieri nel valore traslato 'fare il grande, il prepotente' (es. 12a), grosseggiare, sempre con valore traslato 'fare il grosso, comportarsi da vanaglorioso', attestato nell' Ottimo Commento della Commedia (es. 12b), e infine largheggiare, anch'esso nei valori traslati e lessicalizzati 'essere largo $\rightarrow$ essere generoso' (12c) e 'stare largo $\rightarrow$ entrare comodamente' (12d):

(12a) Un mercennaio intende a grandeggiare,

e pòggiavi sì smisuratamente,

che sofferire già nol può la gente,

veggendol così forte vaneare (Angiolieri, Rime CXXII, vv. 1-4)

(12b) altri, di paura leporina, con la parola grosseggiando mostrano uno ardire di leone

(Ottimo Commento della Commedia, Purgatorio, XI, proemio)

(12c) ed amor ed onor, padre, donare

e cavallaria nova, u' par depona

el piò avar avarizia e largheggiare

pena allora e auro in pregio dona (Guittone, Rime CCXXVII, vv. 5-8)

(12d) il detto Messer Giovanni [...] essendo largheggiato d'andare a ccacciare e uccellare a sua volontà (Filippo Villani, Cronica 76)

È interessante osservare che in nessuno dei tre casi l'aggettivo che costituisce la base del derivato verbale mantiene il valore proprio di aggettivo denotante dimensione fisica. Lo stesso si dirà per il verbo alteggiare, derivato dall'aggettivo alto nello specifico valore traslato riferito all'attitudine di porsi "in alto":

(13) Tu poi intendi chosì di quell'altre: chè quanto a queste cose non è grande isgualglianza: $e$ sse ben pensi, quanto ad alteggiare, vanne abendue di suo nome in pare (Francesco da Barberino, Reggimento e costumi di donna $\mathrm{V}, 5$ )

Lo stesso vale per il verbo altoreggiare, attestato una sola volta nelle Rime di Chiaro Davanzati, dove la base lessicale altore- presuppone un incrocio con la base altero- di alterigia.

Vi sono poi derivati da aggettivi denotanti qualità cosiddette inerenti. Il verbo amareggiare, per es., presumibilmente derivato dal lat. amarizāre, attestato in un trattato di medicina medievale attribuito a Plinio Valeriano, è presente nei testi dell'italiano antico sia nel significato intransitivo 'divenire amaro' (es. 14a), sia in quello transitivo 'rendere amaro $\rightarrow$ addolorare' (14b), in cui peraltro l' aggettivo non ha più valore di qualità inerente:

(14a) A l'aire claro è vista ploggia dare,

ed a lo scuro rendere clarore;

e foco arzente ghiaccia diventare,

e fredda neve rendere calore;

e dolze cose molto amareare,

e de l'amare rendere dolzore (Giacomo da Lentini, Sonetti XXVI, vv. 1-6) 
(14b) quest'ho tormento disperato e fero,

che strugg'e dole e 'ncende ed amareggia (Cavalcanti, Rime XXXIV, vv. 13-14)

Con il valore transitivo di 'rendere chiaro $\rightarrow$ mostrare' è attestato anche il verbo chiareggiare, che ha un'unica attestazione nel corpus e che è senza dubbio da mettere in relazione con il provenzale clareiar e il francese antico clareier. Con valore solo transitivo è attestato il verbo aspreggiare nei diversi significati 'trattare aspramente' (con oggetto animato) e 'rendere aspro $\rightarrow$ duro' (con oggetto anche inanimato, per es. le pene). Anche in questo caso, il processo derivazionale non interessa il valore di proprietà inerente dell'aggettivo aspro ma piuttosto qualità astratte, non-inerenti. Lo stesso si dirà per il verbo caldeggiare, che, seppur derivato dall'aggettivo caldo, si correla ad uno solo dei significati dell'aggettivo, che non è appunto quello cosiddetto di qualità inerente: 'appassionato'. Nella Cronica di Matteo Villani, sono presenti tre attestazioni di forme di caldeggiare, sempre con il valore di 'essere caldo per $\rightarrow$ appoggiare, sostenere'. Vi è infine il verbo graveggiare con due attestazioni nel corpus del TLIO, una intransitiva nel significato 'esser grave' (es. 15a), l'altra transitiva nel significato 'rendere grave $\rightarrow$ appesantire, affliggere' (es. 15b):

(15a) onde quando la mano, overo lo piede, graveggia in giù (Gregorio d'Arezzo, Fiori di medicina)

(15b) guarda s'amore a morte s'appareggia. Ché l'a! dimostra doglia che graveggia, e mor a morte è dritta entenzione (Federico dall' Ambra, Rime II, vv. 5-6)

Vi sono infine i derivati da aggettivi designanti qualità cosiddette non inerenti. Nella maggior parte dei casi i derivati presentano il valore 'fare il x, comportarsi xmente, in maniera x' (dove x è l'aggettivo-base), per es. amorevoleggiare 'essere amorevole, comportarsi amorevolmente', boneggiare 'fare il buono $\rightarrow$ farsi bello di qualcosa', cattiveggiare 'comportarsi da cattivo $\rightarrow$ vivere nell'indolenza', corteseggiare 'comportarsi in modo cortese', follaneggiare 'comportarsi da folle', folleggiare 'comportarsi da folle', gentileggiare 'comportarsi da gentile, cioè da gran signore', grameggiare 'comportarsi in maniera grama $\rightarrow$ darsi pena', matteggiare 'comportarsi da matto', pazzeggiare 'fare il pazzo, compiere atti bizzarri', piacevoleggiare 'fare il piacevole $\rightarrow$ motteggiare, dire battute scherzose', pigreggiare 'comportarsi pigramente', primeggiare 'fare il primo, mettersi in una posizione superiore', ruffianeggiare 'comportarsi da ruffiano', scioccheggiare 'comportarsi da sciocco', spiacevoleggiare 'comportarsi in maniera spiacevole', vagheggiare 'comportarsi in modo vago $\rightarrow$ guardare con ammirazione', vaneggiare 'fare il vano, dire cose vane', villaneggiare 'fare il villano, comportarsi da villano'. In alcuni casi, dalla base semantica generica 'comportarsi in maniera X' derivano specificazioni ulteriori di significato, connesse spesso a processi di lessicalizzazione, per es. grameggiare 'darsi pena', vagheggiare 'guardare con ammirazione'. Vi è, infine, una manciata di verbi deaggettivali in -eggiare che ricorrono in costruzioni transitive e hanno un valore causativo: falseggiare 'rendere falso $\rightarrow$ ridurre il peso della moneta', francheggiare 'rendere sicuro; rendere libero', 
netteggiare $^{20}$ 'rendere netto, sgombrare' e solenneggiare 'rendere solenne'. Infine, transitivo ma privo di valore causativo è il verbo careggiare 'tenere/avere caro', nel quale è presente lo schema di derivazione morfo-lessicale rintracciabile anche nel

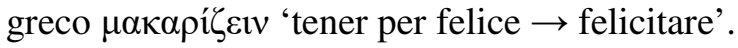

\subsection{Verbi in -eggiare denominali}

Il tipo denominale è di gran lunga il più produttivo, sia nei derivati con-eggiare sia in quelli con -izzare. La tipologia delle strutture sintattiche pertinenti e dei processi derivazionali correlati è molto varia e non si avrà qui modo di discutere tutti i tipi; vedremo quindi quelli più frequenti nel corpus. Una prima distinzione riguarda il tipo di struttura argomentale che il verbo derivato ammette: esso può essere avalente (è il tipo albeggiare $\leftrightarrow$ 'venire/farsi alba'), monovalente (tipologia molto variegata: guerreggiare $\leftrightarrow$ 'fare la guerra', baldanzeggiare $\leftrightarrow$ 'avere baldanza', buffoneggiare $\leftrightarrow$ 'fare il buffone', fortuneggiare $\leftrightarrow$ 'stare alla fortuna', arpeggiare $\leftrightarrow$ 'suonare l'arpa') e bivalente (anche in questo caso tipologia molto variegata: bastoneggiare $\leftrightarrow$ 'colpire con un bastone', danneggiare $\leftrightarrow$ 'fare un danno a', palmeggiare 'tenere nel palmo della mano'). Un'ulteriore suddivisione per i verbi mono- e bivalenti riguarda il tipo di predicazione che il nome che fa da base al derivato ha nella struttura predicativa soggiacente e il tipo di supporto predicativo del nome stesso. Si potrebbe affermare che il suffisso -eggiare esprime i diversi processi predicativi nei quali può entrare il nome che con esso si combina, coerentemente con lo spettro di valori semantici del nome stesso. Un nome di strumento musicale come arpa o citara, con riferimento ad un argomento umano, entrerà preferenzialmente in un processo predicativo correlato al suono dello strumento, per cui arpeggiare e citareggiare valgono 'suonare l' arpa' e 'suonare la citara'. Nel caso dei nomi guerra e baldanza, sempre correlati con un argomento umano, vi sarà riferimento, rispettivamente, ad un'azione ('fare la guerra' $\leftrightarrow$ guerreggiare) e ad un'attitudine ('avere baldanza' $\leftrightarrow$ baldanzeggiare), proprio in funzione del valore semantico specifico dei due nomi. È dunque il nome - con i suoi valori predicativi potenziali - che determina i diversi pattern derivazionali, ragion per cui lo studio della derivazione in -eggiare non può prescindere dall'esame del tipo di nomi che fanno non solo da base lessicale ma anche da scheletro semanticosintattico per il derivato. Problematici sono infine quei denominali che hanno valore sia transitivo sia intransitivo, per es. tiranneggiare 'comportarsi da tiranno'/'trattare in maniera tirannica', attestato in entrambe le costruzioni nel medesimo autore:

(16a) Nel tempo avanti che li fosse chiusa, tiranneggiando fieramente in essa, sanza ricevere o priego o iscusa, tenea la gente sì vilmente oppressa (Boccaccio, Amorosa visione XXXVI 20)

(16b) Oh come fiero li tiranneggiava! (Boccaccio, Amorosa visione XXXVI 25)

In nome dell'unità lessicale, si può ipotizzare un processo di transitivizzazione a partire da un derivato primario intransitivo tiranneggiare 'comportarsi da tiranno', tipo molto produttivo lungo tutta la storia dell' italiano, il quale avrebbe sviluppato

\footnotetext{
${ }^{20}$ Voce di area settentrionale, venez. netizàr, milan. netezàr, piacent. netazàr, da una forma latina non attestata *nitidiāre (GDLI, s.u. nettizzare).
} 
il transitivo tiranneggiare 'trattare in maniera tirannica'. Esempi come questi non sono rari e sono anche attestati nei derivati greci in -íckv e in quelli latini in -issāre/-izāre/-idiāre: uno studio mirato, in prospettiva comparativa, potrebbe far scaturire, oltre ai comuni processi derivazionali, percorsi di lessicalizzazione affini tra le tre lingue.

\subsection{Derivati in -izzare}

Si venga ora brevemente alle forme in -izzare. Anche da un rapido sguardo al lemmario, si nota che nella maggior parte dei casi si tratta di formazioni denominali (con rare eccezioni: avarizzare, egualizzare, sottilizzare), nelle quali peraltro la base lessicale è quasi sempre di origine latina (o greca: per es. alchimizzare, allegorizzare, anatematizzare, canonizzare, dogmatizzare) e talvolta il derivato, prestito dal latino, non è più trasparente in italiano (per es. bombizzare, colafizzare, gargarizzare). Dal punto di vista sintattico-semantico, i valori delle forme in -izzare ricalcano quelli delle forme latine in -izāre e -issāre (e spesso anche delle forme

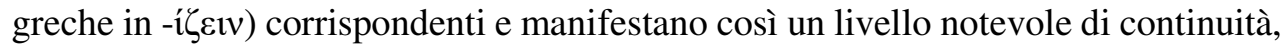
tanto nelle forme quanto nelle funzioni, tra le tre lingue. Tale continuità è frutto dei processi di prestito e calco che hanno interessato nelle diverse epoche greco, latino e italiano: tali processi testimoniano l'elevato grado di prestigio che ebbero, dapprima, il greco nella società romana antica e, a seguire, il latino nell'area italoromanza durante i primi secoli del secondo millennio d.C.

\section{Greco -í̧̧øıv, latino -issārel-izāre/-idiāre e italiano -eggiare e -izzare: osservazioni comparative}

Da una prospettiva squisitamente sincronica sui dati lessicali e testuali delle tre lingue emerge, parallelamente alla linea di continuità cui si accennava poc'anzi, un'importante linea di discontinuità, rappresentata dal latino rispetto a greco antico e italiano. Tale frattura è rilevabile, del resto, solo comparativamente: non c'è quindi da stupirsi se, negli studi dedicati specificamente al latino, essa non emerga e se, soprattutto, le descrizioni disponibili della situazione latina presentino una situazione addirittura rovesciata rispetto a quanto si dirà in séguito, soprattutto in merito alla produttività delle forme pertinenti.

Da un punto di vista quantitativo, infatti, né i types né i tokens delle forme latine in -issāre/-izāre/-idiāre sono confrontabili con quelle greche in -í̧દıv e con quelle in -eggiare e -izzare dell'italiano antico. Lo stesso può dirsi se si osservano i fatti da un punto di vista qualitativo, considerando, per quanto riguarda latino e italiano, sia il rapporto tra prestiti/calchi e neoformazioni, sia la distribuzione testuale delle forme pertinenti nelle due lingue.

Consideriamo intanto i dati quantitativi nelle tre lingue. In latino, il numero di types in -issāre/-izäre/-idiāre ammonta a ca. 120 unità, stando ai risultati della ricerca di Cockburn (2012) che si basa sulle attestazioni del Thesaurus Linguae Latinae e comprende le forme attestate nell'arco di tempo III sec. a.C. - VI sec. 
d.C. ${ }^{21}$ Per i dati dell'italiano antico, come si è detto, abbiamo considerato le ricorrenze del TLIO che include testi dal IX sec. (il primo testo in italiano considerato è l'Indovinello veronese) alla fine del XIV sec. (precisamente il 1375, anno della morte di Boccaccio). Benché si tratti di un arco temporale più breve rispetto a quello documentato dal TLL per le forme latine, in italiano antico è attestato un numero nettamente superiore di forme pertinenti (ca. 240 types in -eggiare e ca. 85 in -izzare) rispetto al latino. Dal punto di vista della distribuzione testuale, tra l'altro, le forme in -eggiare e -izzare ricorrono in tutti i tipi di testi, diversamente da quanto si osserva in latino.

Ancora più sorprendente è la situazione latina se si prendono in considerazione

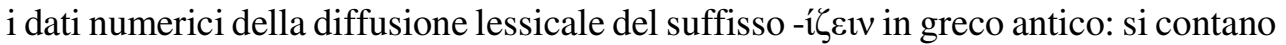
ca. 2700 types (cf. SCHMOLL, 1955 e KRETSCHMER, LOCKER, 1977) per un arco temporale certamente molto ampio, dal sec. VIII a.C. (poemi omerici) al sec. VI d.C. ${ }^{22}$ Il dato meramente numerico diventa più significativo se incrociato con alcune considerazioni relative al tipo di "creazione" lessicale e alla distribuzione testuale delle forme nelle tre lingue. In greco, il suffisso -í̧દlv si combina con qualsivoglia base lessicale: oltre a nomi, verbi, aggettivi, avverbi, numerali, anche sintagmi

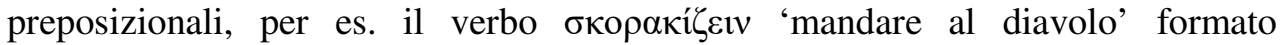

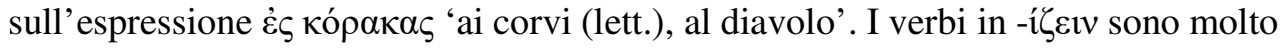
produttivi nelle diverse epoche della storia della lingua greca (non solo del greco antico, peraltro, ma anche del greco moderno), sono attestati in opere letterarie ma anche nella prosa tecnica (per es. nei trattati di medicina: molti i neologismi in Ippocrate e Galeno) e il loro uso non sembra avere alcun tipo di restrizione. ${ }^{23}$ Diversa la situazione in latino, dove la classe dei verbi in -issārel-izāre/-idiāre è composta principalmente da prestiti o calchi dal greco (pochissime le formazioni prive di modelli alloglotti) e la distribuzione nei testi è fortemente condizionata da fattori sociolinguistici dipendenti presumibilmente dalla percezione alloglotta del suffisso $^{24}$ e dalla connotazione diastraticamente bassa (si pensi al greco come lingua degli schiavi) ${ }^{25}$ e diafasicamente orale delle forme in -issāre/-izārel-idiāre.

Non stupisce quindi se la maggior parte delle innovazioni lessicali che presentano i suffissi qui pertinenti sia attestata, da un lato, in Plauto (e in frammenti di teatro arcaico) e, dall'altro, nella letteratura cristiana: due ambiti fortemente influenzati, per ragioni diverse ovviamente, dalla cultura, dal modello e dalla lingua

\footnotetext{
${ }^{21}$ La situazione non cambia molto se si considera la raccolta di dati di DARDANO (2008) che, per il medesimo intervallo temporale, conta un centinaio di types in -issāre e-izāre sulla base del Thesaurus Formarum Totius Latinitatis (sono escluse le forme in -idiāre) o quella di FunCK (1886) che conta 140 forme, includendo anche il latino carolingio.

${ }^{22}$ Nella raccolta di Schmoll (1955) sono presenti anche i lemmi documentati in lessici di epoca posteriore, per es. la Suda, risalente al X sec. d.C., che tuttavia riferiscono forme attestate in opere di epoche precedenti.

${ }^{23}$ Per osservazioni su singole classi di verbi in -í̧દıv si rinvia a TRONCI $(2010,2012,2013)$ e POCCETTI (2014).

${ }^{24}$ Dovuta presumibilmente anche alla presenza del fono rappresentato graficamente prima con $<$ ss $>$, poi con $<\mathrm{Z}>$ e sulla cui realizzazione si rinvia alle osservazioni di MignOT (1969: 330-339) e BiviLLE (1990: 98-136).

${ }^{25}$ Sul bilinguismo greco-latino nella società romana, molto è stato scritto. Per un primo orientamento, cf. Dubuisson (1992a, 1992b), Biville (1992, 2002), AdAms (2003), Mullen (2011).
} 
dei Greci. La lingua latina pare aver agito da filtro rispetto all'espansione delle forme in -issāre/-izäre/-idiāre, impedendo che tali forme permeassero la lingua "alta" dei testi classici e lasciando invece che esse fiorissero, spesso come hápax legómena (è il caso della maggior parte delle forme attestate in Plauto), in testi fortemente connotati dal punto di vista sociolinguistico, in senso diastratico e diafasico, o nelle traduzioni della Bibbia, che appunto risentivano molto del modello greco. Del resto, la maggior parte dei verbi in -izäre che offrono continuità nelle forme e nei valori tra latino e italiano (e, potremmo aggiungere, le lingue romanze in generale) sono quelli attestati nelle traduzioni della Bibbia e nella letteratura cristiana, che presentano molti tokens, a differenza delle forme documentate in altri tipi di testi. Senza dilungarsi sulla questione, ci si limita ad osservare che la produttività delle forme latine in -issārel-izāre/-idiāre è stata presumibilmente molto più elevata di quanto la documentazione latina testimoni e che una prova significativa per questa ipotesi è fornita dai dati dell'italiano antico, in cui è soprattutto la forma del suffisso derivata da regolare mutamento morfo-fonetico, ovvero -eggiare, ad essere la più produttiva, con tipi diversi, peraltro, di basi lessicali (per ulteriori approfondimenti sul tema della produttività delle forme latine si rinvia a TRONCI, 2017).

Come si è rilevato in precedenza, le due forme del suffisso -eggiare e -izzare non mostrano ancora la netta distribuzione funzionale osservabile nell'italiano odierno tra -eggiare, che ricorre preferenzialmente associato a valori di tipo intransitivo-eventivo, e -izzare, specializzato invece nell'espressione di valori transitivo-causativi, come si osserva nella coppia americaneggiare 'comportarsi da americano' e americanizzare 'rendere americano'. Una certa tendenza verso tale distribuzione è rilevabile, tuttavia, già nelle ricorrenze del TLIO ed è presumibilmente da associare ai due diversi percorsi che le forme latine qui pertinenti hanno subito nelle lingue romanze (si veda il §2.4 supra). In italiano antico l'esito del regolare mutamento morfo-fonetico dal latino (-eggiare) è marcato come popolare e orale ed è già molto produttivo con basi lessicali squisitamente italiane, prive di modelli latini. Le forme di prestito dal latino presentano invece il suffisso morfo-foneticamente latineggiante -izzare, hanno basi lessicali per lo più latine e sono meno produttive con basi lessicali italiane.

Dal punto di vista della distribuzione testuale, non si rilevano restrizioni né per le forme in -eggiare né per quelle in -izzare in italiano antico. Numerose forme in -izzare - allegorizzare, anatematizzare, canonizzare, dogmatizzare, esorcizzare, evangelizzare, giudaizzare, idolatrizzare, innizzare, martirizzare, paganizzare, papizzare, profetizzare, sabatizzare, salmizzare, scandalizzare, simonizzare, solennizzare, stimmatizzare, teologizzare, tesaurizzare - sono ricorrenti, come prestiti o calchi dal latino, nelle traduzioni della Bibbia e in testi che si richiamano direttamente alla tradizione cristiana, come già accadeva per molte delle forme latine in -izäre più frequenti, a loro volta prestiti o calchi di originali forme greche

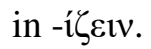




\section{Per concludere}

Prendendo spunto dai dati dell'italiano antico, questo scritto ha proposto qualche riflessione su temi generali che riguardano, da un lato, la derivazione lessicale con riferimento alla categoria del verbo, e, dall'altro, i rapporti diacronici e comparativi delle lingue romanze non solo con il latino ma anche con il greco antico.

Quanto al primo aspetto, l'estrema varietà di rapporti semantici tra base lessicale e derivato - testimoniata dalle diverse parafrasi, spesso associate ad uno stesso derivato, soprattutto nel caso dei denominali - mostra che il processo di "verbificazione", da un lato, è correlato alle potenzialità predicative, spesso implicite, del nome, dall'altro, è dipendente dall'esperienza individuale e sociale, riflessa nella lingua. ${ }^{26}$ Per questa ragione, ci pare che una prospettiva fruttuosa per la ricerca futura possa essere indagare le potenzialità predicative dei nomi che fanno da basi lessicali ai derivati piuttosto che perseguire i tentativi di formalizzazione del modello $L C S$ che ci pare proporre una riscrittura, attraverso un' apposita metalingua, dell'intuizione interpretativa del parlante riflessa nella parafrasi.

Venendo agli aspetti diacronici e comparativi, merita senz'altro sottolineare il dato dell'elevata produttività lessicale delle forme in -íદıı in greco antico, diffuse, senza alcuna restrizione, in ogni tipo testuale (poesia e prosa, sia letteraria sia tecnica) e, per contrasto, la situazione del latino, dove si trovano, sì, patterns derivazionali e valori sintattico-semantici comparabili con quelli dei derivati greci, ma un numero decisamente ridotto di types e tokens (molti lessemi sono hápax legómena). Solo una considerazione comparativa, retrospettiva e prospettiva, consente di descrivere adeguatamente la situazione latina e di argomentare che presumibilmente sono intervenute ragioni normative e spinte puristiche a bloccare la formazione di derivati in -issāre/-izärel-idiāre (cf. TRONCI, 2015, 2017).

L'italiano antico testimonia chiaramente la produttività dei due processi derivazionali, che si collocano, come si è visto, in due ambiti distinti del diasistema: le condizioni della costituzione dei due suffissi, l'uno per trafila fonetica regolare, l'altro per rianalisi da prestiti latini, ha senz'altro giocato un ruolo. Si può anche affermare, del resto, che le condizioni perché una classe di derivati di origine latina sia produttiva in un sistema linguistico romanzo sono ovviamente interne al sistema stesso, come dimostrano, per es., le sorti della classe di verbi francesi in -oyer, che, pur essendo esito di regolare mutamento fonetico dal latino -idiare in area galloromanza, come lo sono nelle rispettive aree l'it. -eggiare e lo sp. -ear, non è produttivo in francese e ricorre soltanto in forme oramai lessicalizzate come flamboyer 'fiammeggiare', rougeoyer 'rosseggiare', verdoyer 'verdeggiare'.

Rimanendo nell'area italo-romanza, sarà interessante studiare come nelle diverse epoche della storia della lingua si siano diffusi nel lessico i derivati qui pertinenti e come essi abbiano contribuito a costituire il lessico di determinati ambiti

\footnotetext{
${ }^{26} \mathrm{Si}$ consideri, per es., il caso dei derivati che predicano la proprietà del colore. Come atteso, molti sono formati su basi lessicali che designano colori (it. rosseggiare, biancheggiare), ma ve ne sono anche altri che hanno basi lessicali che, pur non designando un colore, sono rese pertinenti nel derivato in funzione del colore, per es. lat. chrysizāre 'essere color dell'oro', amethystizāre 'esser del color

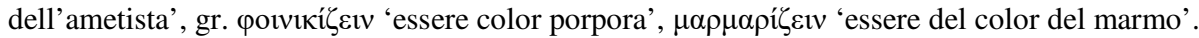


settoriali, per es. della prosa scientifica e tecnica. ${ }^{27}$ Uno studio siffatto offrirebbe spunti interessanti non solo per l'ambito dell'italianistica ma anche, di nuovo, in prospettiva comparativa, nel confronto con il greco antico: come testimonia la

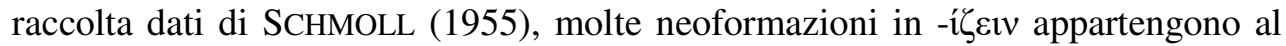
lessico di discipline scientifiche come la medicina, l'architettura, la filologia, fiorite in epoca ellenistica.

\section{BIBLIOGRAFIA}

ADAMS James N. (2003), Bilingualism and the Latin Language, Cambridge, Cambridge University Press.

Alkire Ti, Rosen Carol G. (2010), Romance Languages: A Historical Introduction, Cambridge, Cambridge University Press.

Alvar Manuel, PotTIER Bernard (1983), Morfologia histórica del español, Madrid, Gredos.

ARENA Renato (1965), Contributi alla storia di lat. '-isso', Helikon 5, p. 97-122.

BAUER Laurie (2008), Derivational Morphology, Language and Linguistics Compass 2/1, p. 196-210. https://doi.org/10.1111/j.1749-818X.2007.00045.x

Bergua CAVERo Jorge (2004), Los helenismos del español, Madrid, Gredos.

BertinetTo Pier Marco (2004), Verbi deverbali, in: GROSSMANN Maria, RAINER Franz (éds.), La formazione delle parole in italiano, Tübingen, Niemeyer, p. 465-472.

BIVILLE Frédérique (1990), Les emprunts du latin au grec. Approche phonétique. Tome I: Introduction et consonantisme, Louvain/Paris, Peeters.

BIVILLE Frédérique (1992), Le grec parlé en latin vulgaire. Domaines lexicaux, structures linguistiques d'accueil, in: ILIESCU Maria, MARXGUT Werner (éds.), Latin vulgaire - latin tardif III. Actes du IIIème Colloque international sur le latin vulgaire et tardif (Innsbruck, 2-5 septembre 1991), Tübingen, Max Niemeyer, p. 25-40.

BIVILLE Frédérique (2002), The Graeco-Romans and Graeco-Latin: A Terminological Framework for Cases of Bilingualism, in: ADAMS James N., JANSE Mark, SwAIN Simon (éds.), Bilingualism in Ancient Society, Oxford, Oxford University Press, p. 77-102.

Charitonidis Chariton (2007), Greek -ízo Derivatives: A Conceptual Analysis, Linguistik Online 30/1.

https://www.linguistik-online.net/30_07/charitonidis.html

COCKBURn Olivia C. (2012), Los verbos latinos en -IZARE (-ISSARE, -IDIARE), Madrid, Universidad Autónoma de Madrid.

COCKBURn Olivia C. (2013), Los sufijos verbales -ficare e -izare (-issare, -idiare) y su propagación en el español, in: CASANOVA HERRERO Emili, CALVO RIGUAL Cesáreo (éds.), Actas del XXVI Congreso Internacional de Lingüística y de Filología Románicas, Berlin/Boston, Mouton de Gruyter, vol. 4, p. 505-512.

\footnotetext{
${ }^{27}$ Per es. quello della medicina e delle scienze in generale, cf. DARDANO (2009: 54-55); qualche cenno sulla elevata produttività di -eggiare e -izzare nella prosa, anche scientifica, del Seicento si trova in MigLIORINI (2002 [1960]: 438).
} 
DARDANO Maurizio (2009), Costruire parole. La morfologia derivativa dell'italiano, Bologna, il Mulino.

DARDANO Paola (2008), Contatti tra lingue nel mondo mediterraneo antico: i verbi in -issare/-izare del latino, in: ORIOLES Vincenzo, TOSO Fiorenzo (éds.), Circolazioni linguistiche e culturali nello spazio mediterraneo, Genova, Le Mani Editore, p. 49-61.

DubUISSON Michel (1992a), Le grec à Rome à l'époque de Cicéron. Extension et qualité du bilinguisme, Annales ESC 47/1, p. 187-206.

DUBUISSON Michel (1992b), Le contact linguistique gréco-latin: problèmes d'interférences et d'emprunts, Lalies 10, p. 91-109.

FunCK Anton (1886), Die Verba auf -issare und-izare, Archiv für lateinische Lexikographie und Grammatik 3, p. 398-442.

GDLI = BATTAGLIA Salvatore (éd.) (1961-2002), Grande Dizionario della Lingua Italiana, Torino, UTET.

http://www.gdli.it/

GIULIANI Maria Francesca (2019), L'importanza delle storie lessicali parallele: ancora su pareggio/pileggio 'rotta d'alto mare; tratto di mare aperto', in: Bolatti GuZzo Natalia, TARACHA Piotr (éds.), "And I knew 12 languages". A Tribute to Massimo Poetto on the Occasion of his 70th Birthday, Varsavia, Agade Bis, p. 189-208.

GoICU Viorica (2007), Les verbes avec le suffixe néologique -iza (-isa) en roumain contemporain, in: TROTTER David A. (éd.), Actes du XXIV Congrès International de Linguistique et de Philologie Romanes, Aberystwyth 2004, Tübingen, Niemeyer, tome IV, p. 441-448.

GRANDI Nicola (2008), I verbi deverbali suffissati in italiano. Dai dizionari al web, Cesena/Roma, Caissa Italia Linguistica.

GrossmanN Maria (2004), 5.1. Verbi denominali; 5.2. Verbi deaggettivali, in: GROSSMANN Maria, RAINER Franz (éds.), La formazione delle parole in italiano, Tübingen, Niemeyer, p. 450-465.

GUILBERT Louis (1971), Fondements lexicologiques du dictionnaire: de la formation des unités lexicales, Grand Larousse de la langue française, Paris, Larousse, vol. 1, p. IX-XC.

JACKENDOFF Ray (1991), Parts and boundaries, Cognition 41, p. 9-45.

KRETSCHMER Paul, LOCKER Ernst (1977 [1944]), Rückläufiges Wörterbuch der griechischen Sprache. 3. Unveränderte Auflage. Mit Ergänzungen von Georg Kisser, Göttingen, Vandenhoeck \& Ruprecht.

LA FAUCI Nunzio (2006), Verbi deonomastici e sintassi: sul tipo catoneggiare, in: D’AChILle Paolo, CAFFARElli Enzo (éds.), Lessicografia e onomastica. Atti delle Giornate internazionali di studio (Università di Roma Tre, 16-17 febbraio 2006), Roma, Società Editrice Romana, p. 3-15.

LA FAUCI Nunzio (2010), Anche Madama petrarcheggia?, in: AJELLo Roberto, BerRetToni Pierangiolo, FAnCiUllo Franco, MARotTa Giovanna, MotTA Filippo (éds.), Quae omnia bella devoratis. Studi in memoria di Edoardo Vineis, Pisa, Edizioni ETS, p. 307-315.

LEUMANN Manu (1948), Griechische Verben auf $-i \zeta \varepsilon l v$ im Latein, in: Mélanges de philologie, de littérature et d'histoire anciennes offerts à J. Marouzeau, Paris, 
Les Belles Lettres, p. 371-389 [ristampato in LEumAnN Manu (1959), Kleine Schriften, Zürich/Stuttgart, Artemis, p. 156-170].

LIEBER Rochelle (1998), The suffix -ize in English: implications for morphology, in: LAPOINTE Steven G., BRENTARI Diane K., FARREll Patrick M. (éds.), Morphology and Its Relation to Phonology and Syntax, Stanford, CA, CSLI Publications, p. 12-34.

LIEBER Rochelle (2004), Morphology and Lexical Semantics, Cambridge, Cambridge University Press.

Meul Claire (2009), L'evoluzione dell'infisso latino -idi- nella morfologia verbale romanza: status quaestionis e approfondimenti in base ad una ricerca condotta sul ladino della Val Badia, Rivista di Linguistica 21/2, p. 309-342.

Meul Claire (2013), The Romance reflexes of the Latin infixes -i/esc- and -idi-: restructuring and remodeling processes, Hamburg, Helmut Buske Verlag.

MigLIORINI Bruno (2002 [1960]), Storia della lingua italiana, Milano, Bompiani.

MignOT Xavier (1969), Les verbes dénominatifs latins, Paris, Klincksieck.

MULLEN Alex (2011), Latin and other languages: societal and individual bilingualism, in: ClACKSON James (éd.), A Companion to the Latin Language, Malden, MA / Oxford, Chichester, Blackwell, p. 527-548.

PHARIES David (2002), Diccionario etimológico de los sufijos españoles y de otros elementos finales, Madrid, Gredos.

Plag Ingo (1999), Morphological Productivity: Structural Constraints in English Derivation, Berlin, Mouton de Gruyter.

POCCETTI Paolo (2014), Some thoughts about 'delocutive' verbs in Greek: functions and semantics, in: BARTOLOTTA Annamaria (éd.), The Greek verb Morphology, Syntax, and Semantics. Proceedings of the 8th International Meeting on Greek Linguistics (Agrigento, October 1-3, 2009), Louvain-la-Neuve/Walpole, MA, Peeters, p. 227-252.

RAINER Franz (1993), Spanische Wortbildungslehre, Tübingen, Niemeyer.

ROHLFS Gerhard (1966), Grammatica storica della lingua italiana e dei suoi dialetti. Vol. I: Fonetica, Torino, Einaudi.

ROHLFS Gerhard (1969), Grammatica storica della lingua italiana e dei suoi dialetti. Vol. III: Sintassi e formazione di parole, Torino, Einaudi.

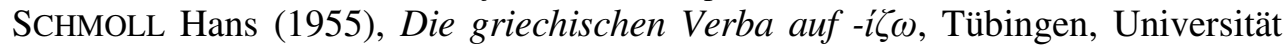
Tübingen.

TEKAVČIĆ Pavao (1972), Grammatica storica dell'italiano, Bologna, il Mulino.

TRONCI Liana (2010), Funzioni, forme, categorie. Una nota su costrutti con verbi in $-i \zeta \omega$, in: Putzu Ignazio, Paulis Giulio, Nieddu Gian Franco, Cuzzolin Pierluigi (éds.), La morfologia del greco tra tipologia e diacronia, Milano, FrancoAngeli, p. 495-511.

TRONCI Liana (2012), Valori differenziali di costrutti con forme verbali in $-i \zeta \omega$, in: LORENZETTI Luca, MANCINI Marco (éds.), Discontinuità e creolizzazione nella formazione dell'Europa linguistica, Roma, Il Calamo, p. 273-289.

TRONCI Liana (2013), Identità di forme, diversità di interpretazioni: $\dot{\varepsilon} \lambda \lambda \eta v i \zeta \omega$, $\beta \alpha \rho \beta \alpha \rho i \zeta \omega$ e la lingua come habitus, in: DE ROgATIS Tiziana, MARRANI Giuseppe, PATAT Alejandro, Russi Valentina (éds.), Identità / diversità, Pisa, Pacini Editore, p. 197-207. 


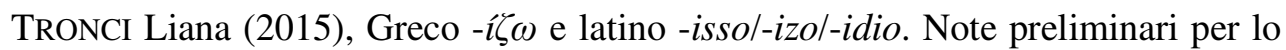
studio di un caso di contatto interlinguistico, in: CONSANI Carlo (éd.), Contatto interlinguistico tra presente e passato, Milano, LED, p. 173-195. http://www.ledonline.it/Il-Segno-le-Lettere/allegati/728-Contatto-

Interlinguistico-Tronci.pdf

TRONCI Liana (2017), Dynamics of linguistic contact. Ancient Greek -ízein and Latin -issāre/-izāre/-idiāre, SKY Journal of Linguistics 30, p. 75-108. http://www.linguistics.fi/julkaisut/SKY2017/SKYJol30_tronci.pdf

VIDOS Benedek Elemér (1939), Storia delle parole marinaresche italiane passate in francese, Firenze, Olschki.

ZAMBONI Alberto (1980-1981), Un problema di morfologia romanza: l'ampliamento verbale in -idio, -izo, Quaderni patavini di linguistica 2, p. 171188.

ZINK Gaston (1986), Phonétique historique du français, Paris, PUF. 


\section{Appendice}

\section{Elenco lemmi da TLIO: 240 derivati in -eggiare}

\begin{tabular}{|c|c|}
\hline abbandeggiare & cattiveggiare \\
\hline accaneggiare & chiareggiare \\
\hline affaneggiare & citareggiare \\
\hline albeggiare & colpeggiare \\
\hline aleggiare & compareggiare \\
\hline allegoreggiare & corneggiare \\
\hline alteggiare & corseggiare \\
\hline altoreggiare & corteseggiare \\
\hline amareggiare & costeggiare \\
\hline amoreggiare & danneggiare \\
\hline amorevoleggiare & dietreggiare \\
\hline anfaneggiare & (dileggiare) \\
\hline annulleggiare & dispareggiare \\
\hline appareggiare & donneggiare \\
\hline appatteggiare & (etimoleggiare) \\
\hline archeggiare & fabuleggiare \\
\hline armeggiare & falleggiare \\
\hline arpeggiare & falseggiare \\
\hline arratteggiare & fanteggiare \\
\hline ascareggiare & favoleggiare \\
\hline aspreggiare & favoreggiare \\
\hline atteggiare & felloneggiare \\
\hline avvileggiare & festeggiare \\
\hline baldanzeggiare & fiammeggiare \\
\hline bamboleggiare & fiancheggiare \\
\hline bandeggiare & filosofeggiare \\
\hline bandoreggiare & folgoreggiare \\
\hline barbareggiare & follaneggiare \\
\hline barcheggiare & folleggiare \\
\hline bastoneggiare & fortuneggiare \\
\hline bastreggiare & francheggiare \\
\hline batteggiare & frascheggiare \\
\hline beffeggiare & frombeggiare \\
\hline berreggiare & fronteggiare \\
\hline berteggiare & fulmineggiare \\
\hline biancheggiare & fumeggiare \\
\hline biondeggiare & galleggiare \\
\hline boccheggiare & gareggiare \\
\hline boneggiar(si) & gentileggiare \\
\hline brandeggiare & ghiottoneggiare \\
\hline buffoneggiare & gialleggiare \\
\hline calcitreggiare & goleggiare \\
\hline caldeggiare & grameggiare \\
\hline calunneggiare & grandeggiare \\
\hline campeggiare & graveggiare \\
\hline camporeggiare & grosseggiare \\
\hline canteggiare & guerreggiare \\
\hline capitaneggiare & guittoneggiare \\
\hline capreggiare & idoleggiare \\
\hline careggiare & impareggiare \\
\hline carreggiare & incarteggiare \\
\hline carteggiare & incorneggiare \\
\hline
\end{tabular}

\begin{tabular}{|c|c|}
\hline indietreggiare & pigreggiare \\
\hline infiammeggiare & pileggiare \\
\hline intorneggiare & polseggiare \\
\hline ladroneggiare & poltroneggiare \\
\hline lampeggiare & pompeggiare \\
\hline langueggiare & pondereggiare \\
\hline largheggiare & primeggiare \\
\hline leccheggiare & principeggiare \\
\hline lenteggiare & profeteggiare \\
\hline lingueggiare & pronteggiare \\
\hline lustreggiare & pulseggiare \\
\hline (malmeggiare) & puttaneggiare \\
\hline maneggiare & questioneggiare \\
\hline manganeggiare & quotidianeggiare \\
\hline marcheggiare & ralleggiare \\
\hline mareggiare & recolteggiare \\
\hline matrigneggiare & ribaldeggiare \\
\hline matteggiare & richieggiare \\
\hline mercanteggiare & ridanneggiare \\
\hline mercatanteggiare & rifiammeggiare \\
\hline molleggiare & ripalleggiare \\
\hline moreggiare & roncheggiare \\
\hline mormoreggiare & rosseggiare \\
\hline motteggiare & ruffianeggiare \\
\hline naveggiare & rumoreggiare \\
\hline netteggiare & salmeggiare \\
\hline noleggiare & sbandeggiare \\
\hline ombreggiare & scalcheggiare \\
\hline ondeggiare & scalpeggiare \\
\hline onesteggiare & schiaffeggiare \\
\hline ormeggiare & scioccheggiare \\
\hline orticheggiare & scorneggiare \\
\hline osteggiare & scorseggiare \\
\hline padroneggiare & scotteggiare \\
\hline paleggiare & scrupoleggiare \\
\hline palmeggiare & sermoneggiare \\
\hline pareggiare & setteggiare \\
\hline pargoleggiare & signoreggiare \\
\hline parteggiare & simoneggiare \\
\hline pasqueggiare & soleggiare \\
\hline passeggiare & solenneggiare \\
\hline pasteggiare & someggiare \\
\hline patteggiare & sorteggiare \\
\hline pavoneggiare & sottorosseggiare \\
\hline pazzeggiare & spaleggiare \\
\hline pennelleggiare & spereggiare \\
\hline penseggiare & sperteggiare \\
\hline pettoreggiare & spesseggiare \\
\hline piacevoleggiare & spiacevoleggiare \\
\hline piateggiare & spinteggiare \\
\hline pazzeggiare & toccheggiare \\
\hline pignoreggiare & storieggiare \\
\hline
\end{tabular}


Spunti per una descrizione dei verbi in -eggiare $e$-izzare: I dati dell'italiano antico in prospettiva diacronica e comparativa

stormeggiare strazieggiare svergheggiare svillaneggiare taglieggiare taverneggiare temoleggiare temporeggiare

tristeggiare
tronconeggiare
truffeggiare
usureggiare
vagheggiare
vaneggiare
vanteggiare
veleggiare

venteggiare verdeggiare vergheggiare vettureggiare vezzeggiare vigoreggiare villaneggiare volteggiare

\section{Elenco lemmi da TLIO: 85 derivati in -izzare}

adumanizzare
agonizzare
alchimizzare
allegorizzare
anatematizzare
anatemizzare
anatomizzare
(annizzare)
aramatizzare
aristotelizzare
armonizzare
aromatizzare
autorizzare
attorizzare
avarizzare
barbarizzare
bombizzare

canonizzare
cautelizzare
cauterizzare
citarizzare
clisterizzare
colafizzare
dogmatizzare
egualizzare
esorcizzare
etimologizzare
evangelizzare
favolizzare
favorizzare
festizzare
filosofizzare
gargarizzare
garrizzare

giudaizzare

guagnelizzare

idolatrizzare

impalizzare

innizzare

intesaurizzare

intizzare

intronizzare

martirizzare

martorizzare

mercantizzare

metaforizzare

moralizzare

notomizzare

organizzare

paganizzare

papizzare

particolarizzare
patrizzare
pessarizzare
petizzare
poetizzare
polverizzare
preconizzare
prodigalizzare
proemizzare
profetizzare
reumatizzare
rivolgarizzare
sabatizzare
salmizzare
scandalizzare
senapizzare
sgotizzare

signorizzare

sillogizzare

simonizzare

sincopizzare

solennizzare

sottilizzare

spermatizzare

spolverizzare

stercorizzare

stimmatizzare

teologizzare

tesaurizzare

tirannizzare

tortizare

umanizzare

vangelizzare

volgarizzare 